\title{
Keratocyst Odontogenic Tumor (KCOT): Treatment Methods at the Second Affiliated Hospital of Jiamusi University
}

\author{
Nyimi Bushabu Fidele ${ }^{1}$, Feng Duan1', Em Kalala Kazadi², Jian Guan¹, \\ Mantshumba Milolo Augustin ${ }^{3}$, Yuwen Zhou ${ }^{4}$ \\ ${ }^{1}$ Department of Oral and Maxillofacial Surgery (Unit I), Second Affiliated Hospital of Jiamusi University, \\ Jiamusi, China \\ ${ }^{2}$ Stomatology and Maxillofacial Service (Unit of Periodontology Surgery), Affiliated Hospital of Kinshasa \\ University, Kinshasa, DR. Congo \\ ${ }^{3}$ Prosthodontics and Orthodontics Service, Affiliated Hospital of Kinshasa University, Kinshasa, DR. Congo \\ ${ }^{4}$ Department of Prosthodontics, Second Affiliated Hospital of Jiamusi University, Jiamusi, China \\ Email: *fidelenyimi@yahoo.fr
}

Received 27 August 2015; accepted 7 November 2015; published 10 November 2015

Copyright (C) 2015 by authors and Scientific Research Publishing Inc.

This work is licensed under the Creative Commons Attribution International License (CC BY). http://creativecommons.org/licenses/by/4.0/

(c) () Open Access

\section{Abstract}

Many studies have been done about the treatment of KCOT, but the recurrence rate still remains high despite the combination of surgical techniques used, associated with or without the use chemical solutions such as Carnoy's solution or Cryosurgery. Currently, there is no consensus concerning the therapeutic choice for the KCOT. The purpose of this study was to evaluate the different treatment methods used for the management of KCOT in the Second Affiliated Hospital of Jiamusi University and the results obtained from those treatment methods. Settings and Design: Clinical study was done at the Second Affiliated Hospital of Jiamusi University. Materials and Methods: Analytical retrospective review of 63 cases treated surgically from 2009 to 2015 at the Maxillofacial and Oral Surgery Department of the Second Affiliated Hospital of Jiamusi University. All cases diagnosed as KCOT and registered in the archives were used. The level of statistical significance was set at $P<0.05$. Results: Of the 63 cases, $55.56 \%$ were male and $44.44 \%$ female. The sex ratio was 1.25 in favor of male and the average age was 42 years. $81 \%$ of the lesions were located in the mandible. of 40 cases treated by enucleation with peripheral ostectomy, curettage and electric cauterization with application of $2 \%$ iodine tincture, no recurrence was found. 0 f 20 cases treated by the same surgical method but without the application of $2 \%$ iodine tincture, recurrence was found in 3 cases $(4.76 \%)$. 3 cases treated by resection had no recurrence. Significant relation between recurrence and type of treatment was found $(P<0.05)$. Conclusion: In our series, those cases that were treated by combination of surgical methods used with $2 \%$ iodine tincture

\footnotetext{
${ }^{*}$ Corresponding author.
}

How to cite this paper: Fidele, N.B., Duan, F., Kazadi, E.K., Guan, J., Augustin, M.M. and Zhou, Y.W. (2015) Keratocyst Odontogenic Tumor (KCOT): Treatment Methods at the Second Affiliated Hospital of Jiamusi University. Open Journal of Stomatology, 5, 251-258. http://dx.doi.org/10.4236/ojst.2015.511031 
showed an "improvement" in the reduction of recurrence, suggesting a future question to be examined in experimental study is to test the effectiveness of $2 \%$ iodine tincture.

\title{
Keywords
}

\author{
Keratocystic Odontogenic Tumors, Keratocyst, Odontogenic Keratocyst, Iodine Tincture
}

\section{Introduction}

The keratocystic odontogenic tumor (KCOT) is defined, according to the World Health Organization (WHO) as a benign tumor uni- or multicystic, intraosseous tumor of odontogenicorigin, with a characteristic lining of parakeratinized stratified squamous epithelium and potential aggressive, infiltrative behavior [1]. It may be solitary or multiple and the latter is usually one of the stigmata of the inherited naevoid basal cell carcinoma syndrome (NBCCS). Its first description was made by Philipsen in 1956. The KCOT has classified odontogenic cyst. Since 2005, WHO has classified them such as benign keratocystic odontogenic tumors due to their biologic aspect [1]-[3]. KCOTs account for $10 \%-14 \%$ of all cysts of the jaws, and may occur at any age, but usually during the second and third decades of life. It's frequently localized in the posterior region of the mandible and has higher occurrences in women than in men [3]-[6].

Although these are benign tumors, the recurrence rate of KCOT has been set at of $4 \%$ - $70 \%$ after the treatment depending on the surgical techniques used, and recurrences are more common during the first 4 years post surgery [7]-[9]. However, the enucleation surgical method that traditionally was used to treat the KCOT is not sufficient. And the treatment that aims to prevent recurrences is questionable due to deletion of a large amount of bone surrounding the lesions which generate significant risks (nerve damages, loss of facial form). Many current studies have been done about the treatment of KCOT. However, the results from these studies have shown that despite the combination of surgical techniques used, associated with or without use of chemical solutions such as Carnoy's solution or Cryosurgery, the recurrence rate still remains high, ranging according to the results of works, as done in University of Bern in Germany (17\% - 56\%), Brazil (13\% - 50\%), China (15\%), La Paz in Spain (25\%) and the University of Tunisia (41\%) [4] [10]-[13]. Currently there is no consensus concerning the therapeutic choice for the keratocyst odontogenic tumor. Thus, there is uncertainty about the best treatment options chosen that can permit to establish the effectiveness of the available treatments, to give a satisfactory prognosis for short or long term duration after tumor removal. Hence, in order to evaluate the different treatment methods available for the management of KCOT in the Second Affiliated Hospital of Jiamusi University we have chosen this study.

The purpose of this paper was to conduct a retrospective study of KCOTs cases treated over a period of 6 years at the Department of Oral and Maxillofacial Surgery at Second Affiliated Hospital of Jiamusi University, through the analysis of clinical, epidemiological aspects and with special emphasis on the therapeutics modalities of the data chosen and its effect.

\section{Materials and Methods}

Analytical retrospective review of 63 cases of KCOTs treated surgically from 2009 to 2015 in maxillofacial and oral surgery department at the second affiliated Hospital of Jiamusi University were carried out. The medical files of all the patients treated for tumor of maxilla and mandible were consulted and followed up, hospitalized or not, at the oral and maxillofacial surgery department, Unit I and II of the second affiliated Hospital of Jiamusi University, and included in the population study of our research. In the period chosen for this present study of 6 years, 487 files of patients consulted were registered. Among them, 63 files of patients' filled in the criteria. The inclusion criteria were, the patients who were surgically treated, histologically diagnosed as KCOTs and those followed up from 3 months till 5 years post-treatment. The KOCT associated to Gorlin-Goltz syndrome were excluded. Demographic data collected included age and gender. This information was collected through research in the register book, using keratocyst, keratocystic odontogenic tumor, odontogenic cyst or odontogenic keratocyst as the main search terms.

The research protocol was submitted to the ethical committee of the second affiliated hospital of Jiamusi 
University for Approbation. It is after informed consent of ethical committee that we have collected the data; the ethics were judiciously considered. The data collected from the case files were, medical form numbers, address of patients, operation dates, telephone numbers, patients' antecedents, radiologic and clinical signs, histological diagnosis, prophylactic and curative treatments and follow ups. The localization of the tumors was differently divided into maxillary, either anterior or posterior regions (incisor-canine or premolar-molar). The mandible itself was divided into different anatomic areas: chin, body of mandible, angle of mandible, angle and ramus of mandible, and ramus and condylar area. The radiographic findings described included, a simple or multiple localization with or without the presence of an impacted tooth. The histological type of KCOT finding was recorded.

The treatment options included resection, enucleation with curettage and/or peripheral ostectomy, electric cauterization of cyst cavity with or without the use of $2 \%$ iodine tincture. Application of $2 \%$ iodine tincture solution was combined with the other treatment. The treatment options for the patients were randomly chosen. All treatment techniques used, follow-up period, any complication during the treatment time, localization and management of recurrence was systematically recorded. The time of recurrence was calculated as the number of months or years between the surgical treatment and the biopsy of the recurrence. Surgical management of the recurrence was classified in the same groups as for treatment of the primary lesions.

The statistical analysis data was done with SPSS version 20. And the association between interest variables such as sex, size, localization, radiological image, symptoms, histological types, treatment used and recurrence were searched. The statistic tests used were the Chi-square test, the Fisher exact test and Student t test. Qualitative data were described by absolute and relative frequencies, but Quantitative data were described by mean or the median. The significance level was set at $P<0.05$.

\section{Results}

In total of 63 cases, $55.56 \%$ were the male and $44.44 \%$ were female (Figure 1). The average age in this present study was 42 year and the gender ratio was 1.25 . The age groups of 21 - 30 and 51 - 60 years were most affected by KCOTs with $38.1 \%$, and $22.2 \%$ respectively.

Clinically, more than half of KCOTs were asymptomatic (52.4\%) and $47.6 \%$ were symptomatic. Symptoms included pain, tumefaction, hypoesthesia; trismus and infection were differently represented (Figure 2). The most frequent radiological image of KCOTs was unilocular lesions (73.02\%) and 31.17\% associated with an impacted tooth.

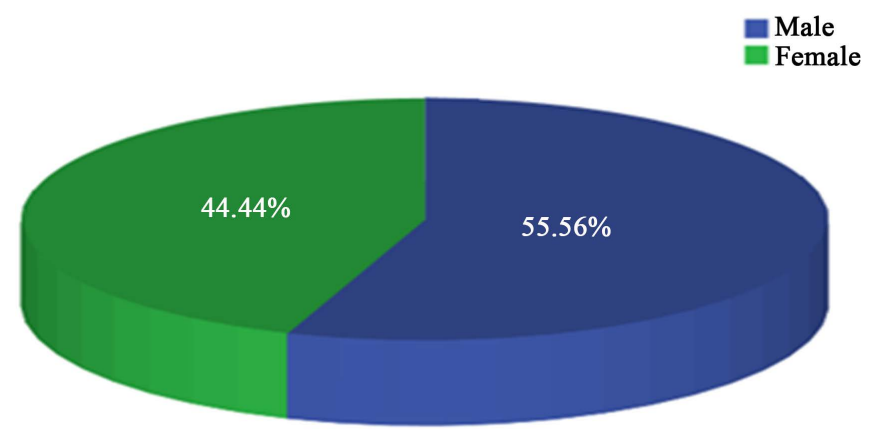

Figure 1. Repartition of KOCs per sex.

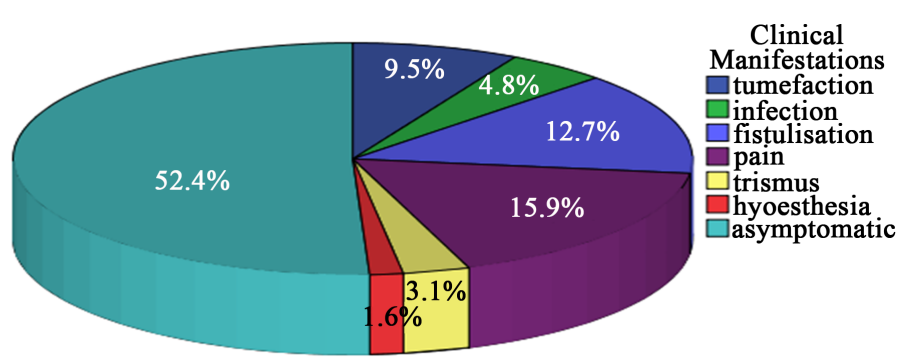

Figure 2. Clinical manifestations of KCOTs. 
The majority of the lesions (81\%) were located in the mandible (Table 1 and Table 2), mostly in the mandible body (36.51\%) followed by the angle of the mandible (20.63\%), Angle and Ramus (15.87\%), chin (6.35\%) and Ramus-condyle (1.59\%). Occurrence in the maxilla was $19.05 \%$, and the lesion was more located in posterior region with $14.25 \%$. More than half of KCOTs had a diameter greater than $4 \mathrm{~cm}$ (52.4\%), followed by a 2 $4 \mathrm{~cm}$ diameter (33.3\%) and diameters less than $2 \mathrm{~cm}(14.3 \%)$.

The most frequent treatment methods used was enucleation associated with curettage or peripheral ostectomy (95.24\%). Enucleation treatment method associate to adjuvant treatment use 2\% iodine tincture and cauterization cystic cavity were most frequently used (63.49\%), and in 31.75\% of cases the same treatment was undertaken, but without the use of iodine tincture. Three cases were treated by resection only method (4.76\%) (Table 3). The treatment was associated with or without tooth extraction.

After a mean follow-up period of 3 years, 40 cases treated by enucleation with curettage, peripheral ostectomy, and electric cauterization of cyst cavity associated with $2 \%$ iodine tincture, and in 3 cases treated by resection only method, no recurrence was found. In 20 cases treated by enucleation associated with curettage, peripheral ostectomy, and electric cauterization without use of $2 \%$ iodine tincture, recurrence was found in 3 cases (4.76\%) (Table 3). All of the recurrences found were in the mandible, and the mandible angle and Angle-Ramus region are the part where the recurrence has been observed with $3.17 \%$ and $1.59 \%$ respectively (Table 4 ). The age distribution of recurrence was similar to the primary cases, showing a peak in the second decade followed by the five decade of life. No significant association was seen between histologic types of KCOTs, symptoms cases and recurrence $(P>0.05)$. The recurrence of the KCOTs was found on $2^{\text {nd }}$ (1case) and $3^{\text {rd }}$ (2cases) year post-treatment (Figure 3).

A significant association was seen between recurrence and sex, multilocular appearance, diameter of KCOT $(P<0.05)$. Statistical analysis showed a significant relation between recurrence and the type of treatment used. Regarding the anatomical location, area lesions involving the mandibular angle showed a predominance recurrence rate (3.17\%) than lesions involving mandibular angle-ramus (1.59\%).

Table 1. Distribution of KCOT on the maxilla and mandible.

\begin{tabular}{cccc}
\hline & Maxilla & Mandible & Total \\
\hline Number & 12 & 51 & 63 \\
Percentage (\%) & 19.0 & 81.0 & 100 \\
\hline
\end{tabular}

Table 2. Distribution of KCOT according to maxilla and mandible region.

\begin{tabular}{cccc}
\hline Jaws & Regions & Number of KCOTs & Percent \\
\hline Mandible & Chin & 4 & 6.35 \\
& Mandible body & 23 & 36.51 \\
& Angle of mandible & 13 & 20.63 \\
& Angle and ramus & 10 & 15.87 \\
Raxillary & Ramus and condylar & 1 & 1.59 \\
& Anterior & 3 & 4.76 \\
Total & Posterior & 9 & 14.29 \\
\hline
\end{tabular}

Table 3. Distribution of recurrence according to treatment methods used.

\begin{tabular}{cccc}
\hline & $\begin{array}{c}\text { En-Cu-Po + Caut 2\% } \\
\text { of iodine tincture }\end{array}$ & $\begin{array}{c}\text { En-Cu-Op + Caut } \\
\text { without iodine tincture }\end{array}$ & Resection \\
\hline Number & $40(63.49 \%)$ & $20(31.75 \%)$ & $3(4.76 \%)$ \\
Recurrence & $0(0 \%)$ & $3(4.76 \%)$ & $0(0 \%)$ \\
\hline
\end{tabular}

En = enucleation; $\mathrm{Cu}$ = curettage; $\mathrm{Op}$ = peripheral ostectomy; Caut = electric cauterization and $\%=$ percent. 


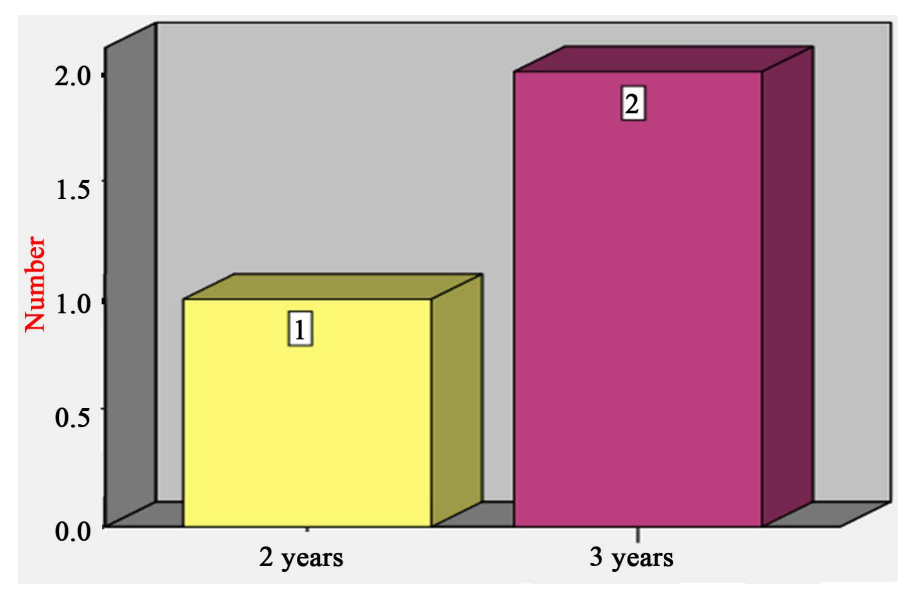

Figure 3. Association timing and recurrence.

Table 4. Distribution of recurrence based on the mandible regions.

\begin{tabular}{cccc}
\hline Regions & Number of KCOTs & Recurrence & Percent \\
\hline Chin & 4 & 0 & 0 \\
Mandible body & 23 & 0 & 0 \\
Angle of mandible & 13 & 2 & 3.17 \\
Angle and Ramus & 10 & 1 & 0 \\
Ramus and condylar & 1 & 0 & 4.59 \\
Total & 51 & 3 & 0 \\
Missing & 12 & 0 & 4.76 \\
Total & 63 & 3 & 4.76 \\
\hline
\end{tabular}

\section{Discussions}

Regarding gender and age distribution, our findings showed a mean age of 42 years and the male presented a slight predominance (55.56\%), with sex ratio of 1.25 in favor of males. Those results were similar to those of other studies [4] [11] [14]. Age groups between 21 - 30 years and 51 - 60 years were the most affected in this present study (38.1\%) and (22.2\%) respectively which are in agreement with those found by other authors [10], but distinct from those found by Leite TC age groups of $11-20$ and 31 - 40 years were the most affected, followed by 21 - 30 years [11]. This difference may be explained by the fact that the methodologies and the criteria of selection were different to those chosen in our series. In this study, the KCOTs associated to Corlin-Goltz syndrome were excluded while that Leite TC and Shear studies included them. According to the current studies, in patients with Gorlin-Goltz syndrome, the diagnosis of KCOTs is done in the early period of life (10 - 20) years of age [4] [15]. This could justify the age groups of $11-20$ years having a high prevalence of KCOTs in their studies [11] [16].

Clinically, 52.4\% of KCOTs in present study were asymptomatic which are similar to those found by others authors, who stipulate that $50 \%$ to $62 \%$ of cases of KCOTs are asymptomatic [4] [10]. On the contrary, our results are in discord with those found by other authors, where more than half of KCOTs was symptomatic at 53.8\% [11]. That may be justified by fact that, our study includes only the sporadic KCOTs, but the others studies included the KCOTs associated to Gorlin-Goltz syndrome, who according to the others studies presented more clinical manifestations than sporadic KCOTs [15] [17].

This discovery explain the fact that the KCOTs are essentially in jaw and $81 \%$ of cases are located in mandible that is constituted with a compact bone. The space of osseous marrow serve as slide of guidance in tumor 
growth and recessed in bone till the moment where the tumor volume become unbearable that its expansion may give the visible clinical manifestations. The radiologic appearance was frequently unilocular (73.02\%), associated with an impacted tooth (31.17\%), which concurs to other studies [3] [18].

The most KCOTs occurred in the mandible (80\%), which is similar to those results of other studies [4] [5]. The most common area at the mandible was the mandible body, followed by the angle and ramus. Similar results were found by the others authors [3] [19] [20]. About the regions of these lesions, the data from literature seems more contradictory. For the first group of authors found that KCOTs were more located in the mandible body, followed by the angle [3] [19]. However the second group found KCOTs were in the mandible body, but followed by Ramus [21] [22]. On the contrary the third groups of authors had opposite results; the mandibular angle being more affected followed by the Ramus [16]. But, our results correspond with those found by the first group of authors [4].

Results of the recurrent study shows that the recurrence rate varies between $4 \%-70 \%$ according to surgical techniques used and the adjuvant treatment associated [7] [8] [23]. In our series, the recurrence rate observed was $4.76 \%$ after a mean follow-up period of 3 years. The significant association was found between sex and recurrence $(P<0.05)$, which are similar with other studies and the recurrence rate did correlate with KCOT size [14]. The recurrences were essentially observed in the mandible, angle and ramus regions were affected with $3.17 \%$ and $1.59 \%$ respectively. This result concurs with those found by other authors [12]. The Angle of mandible and Ramus constitute regions where the vision atoperative field is reduced, which causes the incomplete removal of tumor, thus subsequent recurrences factor. A significant association between multilocular radiologic image and recurrence has been established $(P<0.05)$, which is in agreement with others studies [17]. No significant association was found between the daughter cysts and recurrence $(P>0.05)$, though some others have reported [3]. In fact it should be explained that in this present study, the recurrence rate was low, (4.76\%) in a sample of 63 cases of KCOTs, whilst the other author with a sample of 55 cases of KCOTs, 14 cases of recurrences were observed (25.45\%) [4].

It was found by the current study that in the treatment of KCOTs using resection, marsupialization, enucleation with curettage, peripheral ostectomy, showed a recurrence rate situated between $17 \%$ - 56\%, when one associates the Carnoy's solution, the rate was found to decreases as $1 \%-8.7 \%$ and it drops to $0 \%$ when the resection is done [10] [24]. On the contrary, it was found by other studies that the KCOTs presented recurrence rate differently: $11 \%, 15 \%, 25 \%, 29 \%, 50 \%$ according to surgical techniques used, such as enucleation associated with curettage, peripheral otectomy, marsupialization, resection and with or without the Carnoy's solution or Cryosurgery [4] [10] [12] [25] [26].

Butin this present study, no recurrence was found in 40 cases of KCOTs treated by enucleation with curettage, peripheral ostectomy, and electric cauterization cavity cyst, associated with $2 \%$ of iodine tincture. In contrary, the 20 cases treated with the same treatment, without $2 \%$ iodine tincture, the recurrence was found of 3 cases (4.76\%). The explanation should be in fact that the combinations of surgical techniques used were not the same than others studies and Carnoy's solution and/or cryosurgery were replaced by $2 \%$ iodine tincture in our study.

Concerning the recurrence time, our finding of the recurrence time showed, one case was (1.59\%) and 2 cases (3.17\%) during the $2^{\text {nd }}$ and $3^{\text {rd }}$ year post-treatment. These results were similar to those found by Browne, Foresell et al. [3] [13]. Surgical management of the recurrence was classified in the same groups as for treatment of the primary lesions. Note was also made of any reconstructive technique employed.

The limitations of this study:

-It is an analytical, retrospective study done in the university for research purpose, there may be transcription bias during the typing of the different medical records,

-We expect to have a larger size of sampleand from different hospitals than used in this study.

\section{Conclusion}

In our series, those cases treated by combination surgical methods with use of $2 \%$ iodine tincture show an improvement in the reduction of recurrence, suggesting a future question that needs to be examined is experimental study to test the efficiency of $2 \%$ iodine tincture. Resection of the jaws, treatment that aims to prevent the recurrences of KCOT, is questionable due to deletion of a large amount of bone surrounding the lesion which significant risks (nerve damages, loss of facial form). Unless resection is necessary, it seems appropriate to use enucleation associated with use of $2 \%$ iodine tincture. 


\section{Acknowledgements}

No financial grants, industrial links or affiliation.

\section{Conflict of Interest}

The authors declare no conflict of interest regarding the publication of this paper.

\section{References}

[1] Barnes, L., Everson, J.W., Reichart, P. and Sidransky, D. (2005) Pathology and Genetics of Head and Neck Tumors: WHO Classification of Tumours Series. IARC Press, Lyon, 284-306.

[2] Vered, M., Peleg, O., Taicher, S. and Buchner, A. (2009) The Immunoprofile of Odontogenic Keratocyst (Keratocystic Odontogenic Tumor) That Includes Expression of PTCH, SMO, GLI-1 and bcl-2 Is Similar to Ameloblastoma but Different from Odontogenic Cysts. Journal of Oral Pathology \& Medicine, 7, 597-604. http://dx.doi.org/10.1111/j.1600-0714.2009.00778.x

[3] Kramer, I.R., Pindborg, J.J. and Shear, M. (1992) The WHO Histological Typing of Odontogenic Tumors: A Commentary on the Second Edition. Cancer, 12, 2988-2994. http://dx.doi.org/10.1002/1097-0142(19921215)70:12<2988::AID-CNCR2820701242>3.0.CO;2-V

[4] Sánchez-Burgos, R., González-Martín-Moro, J., Pérez-Fernández, E. and Burgueno-Garcia, M. (2014) Clinical, Radiological and Therapeutic Features of Keratocystic Odontogenic Tumors: A Study over a Decade. Journal of Clinical and Experimental Dentistry, 3, e259-e264. http://dx.doi.org/10.4317/jced.51408

[5] González-Alva, P., Tanaka, A., Oku, Y., Yoshizawa, D., Itoh, S., Sakashita, H., Ide, F., Tajima, Y. and Kusama, K. (2008) Keratocystic Odontogenic Tumor: A Retrospective Study of 183 Cases. Journal of Oral Science, 2, 205-212. http://dx.doi.org/10.2334/josnusd.50.205

[6] Habibi, A., Saghravanian, N., Habibi, M., Mellatit, E. and Habibi, M. (2007) Keratocystic Odontogenic Tumor: A 10-Year Retrospective Study of 83 Cases in an Iranian Population. Journal of Oral Science, 3, 229-235. http://dx.doi.org/10.2334/josnusd.49.229

[7] Sharif, F.N.J., Oliver, R., Sweet, C. and Sharif, M.O. (2010) Interventions for the Treatment of Keratocystic Odontogenic Tumors (KCOT Odontogenic Keratocysts (OKC)). Cochrane Database of Systematic Reviews, 9, Article ID: CD008464. http://dx.doi.org/10.1002/14651858.CD008464.pub2

[8] Nakamura, N., Mitsuyasu, T., Mitsuyasu, Y., Tabrizi, R., Zkan, B.T. and Dehgani, A. (2002) Marsupialization for Odotogenic Keratocyst: Long-Term Follow-Up Analysis of the Effects and Changes in Growth Characterstics. Oral Surgery, Oral Medicine, Oral Pathology, Oral Radiology \& Endodontology, 5, 543-553. http://dx.doi.org/10.1067/moe.2002.128022

[9] Voorsmit, R.A., Stoelinga, P.J. and Van Haelst, U.J. (1981) The Management of Keratocysts. Journal of Oral and Maxillofacial Surgery, 4, 228-236. http://dx.doi.org/10.1016/s0301-0503(81)80049-5

[10] Bornstein, M.M., Filippi, A., Altermatt, H.J., Lambrecht, J.T. and Buser, D. (2005) Le kératokyste odontogène: Kyste odontogène ou tumeur bénigne? Revue Mensuelle Suisse d'Odonto-Stomatologie., 115, 123-128.

[11] Leite, T.C., Meirelles, J.R.V. and Janimi, M.E.R. (2011) Odontogenic Keratocystic Tumor: A Clinical and Histopathologic Retrospective Study Based on the New WHO Classification. International Journal of Odontostomatology, 5, 227-234. http://dx.doi.org/10.4067/S0718-381X2011000300004

[12] Zhao, Y.F., Wei, J.X. and Wang, S.P. (2002) Treatment of Odontogenic Keratocysts: A Follow-Up of 255 Chinese Patients. Oral Surgery, Oral Medicine, Oral Pathology, Oral Radiology, and Endodontology, 94, 151-156. http://dx.doi.org/10.1067/moe.2001.125694

[13] Chemli, H., Dhouib, M., Karray, F. and Abdelmoula, M. (2010) Les facteurs de risque de récidive des kératokystes odontogéniques des maxillaire. Revue de Stomatologie et de Chirurgie Maxillo-faciale, 111, 189-192. http://dx.doi.org/10.1016/j.stomax.2009.07.011

[14] Myoung, H., Hong, S.P., Hong, S.D., Lee, J.I., Lim, C.Y., Choung, P.H., Lee, J.H., Choi, J.Y., Seo, B.M. and Kim, M.J. (2001) Odontogenic Keratocyst: Review of 256 Cases for Recurrence and Clinicopathologic Parameters. Oral Surgery, Oral Medicine, Oral Pathology, Oral Radiology, and Endodontology, 91, 328-333. http://dx.doi.org/10.1067/moe.2001.113109

[15] Kissi, L., Benyahya, I. and Rifki, A. (2006) Le keratokyste isole: A propos d'un cas. Journal de l'ordre des dentistes du Québec, 43, 321-328.

[16] Shear, M. and Speight, P.M. (2007) Odontogenic Keratocyst of the Oral and Maxillofacial Regions. 4th Edition, Blackwell Munksgaard, Oxford, 6-58. 
[17] Dghoughi, S., Chbiched, S. and Wady, E.I.W. (2009) Une tumefaction gingivale anterieure. Revue de Stomatologie et de Chirurgie Maxillo-faciale, 110, 242-245. http://dx.doi.org/10.1016/j.stomax.2009.06.001

[18] Forsell, K. (1980) The Primordial Cyst. A Clinical and Radiographic Study. Proceedings of the Finnish Dental Society, 76, 129-174.

[19] Morgan, T.A., Burton, C.C. and Quian, F. (2005) A Retrospective Review of Treatment of the Odontogenic Keratocyst. Journal of Oral and Maxillofacial Surgery, 63, 635-639. http://dx.doi.org/10.1016/j.joms.2004.07.026

[20] Köndell, P.A. and Wiberg, J. (1988) Odontogenic Keratocysts. A Follow-Up Study of 29 Cases. Swedish Dental Journal, 12, 57-62.

[21] Oda, D., Rivera, V., Ghanee, N., Kenny, E.A. and Dawson, K.H. (2000) Odontogenic Keratocyst: The Northwestern USA Experience. Journal of Contemporary Dental Practice, 1, 60-74.

[22] Browne, R.M. (1970) The Odontogenic Keratocyst. Clinical Aspects. British Dental Journal, 128, $225-231$.

[23] Hyun, H.K., Hong, S.D. and Kim, J.W. (2009) Recurrent Keratocystic Odontogenic Tumor in the Mandible: A Case Report and Literature Review. Oral Surgery, Oral Medicine, Oral Pathology, Oral Radiology, and Endodontology, 108, e7-e10. http://dx.doi.org/10.1016/j.tripleo.2009.04.030

[24] Hajj, G.E.L. and Anneroth, G. (1996) Odontogenic Keratocysts. A Retrospective Clinical and Histologic Study. International Journal of Oral and Maxillofacial Surgery, 25, 124-129. http://dx.doi.org/10.1016/S0901-5027(96)80057-9

[25] Shear, M. (2002) The Aggressive Nature of the Odontogenic Keratocyst: Is It a Benign Cystic Neoplasm? Part 2. Proliferation and Genetic Studies. Oral Oncology, 38, 323-331. http://dx.doi.org/10.1016/S1368-8375(01)00066-5

[26] Pan, S. and Li, T.J. (2009) PTCH1 Mutations in Odontogenic Keratocysts: Are They Related to Epithelial Cell Proliferation. Oral Oncology, 45, 861-865. http://dx.doi.org/10.1016/j.oraloncology.2009.02.003 